\title{
Non-Blind Deblurring Using Partial Differential Equation Method
}

\author{
Devender Sharma \\ CSE Department \\ HCE,Sonepat, \\ India.
}

\author{
Puneet Sharma \\ CSE Department \\ HCE,Sonepat, \\ India.
}

\author{
Ritu Sharma \\ ECE Department \\ BMIET,sonepat, \\ India.
}

\begin{abstract}
In this paper, a new idea for two dimensional image deblurring algorithm is introduced which uses basic concepts of PDEs... The various methods to estimate the degradation function (PSF is known in prior called non-blind deblurring) for use in restoration are observation, experimentation and mathematical modeling. Here, PDE based mathematical modeling is proposed to model the degradation and recovery process. Several restoration methods such as Weiner Filtering, Inverse Filtering [1], Constrained Least Squares, and Lucy -Richardson iteration remove the motion blur either using Fourier Transformation in frequency domain or by using optimization techniques. The main difficulty with these methods is to estimate the deviation of the restored image from the original image at individual points that is due to the mechanism of these methods as processing in frequency domain .Another method, the travelling wave de-blurring method is a approach that works in spatial domain.PDE type of observation model describes well several physical mechanisms, such as relative motion between the camera and the subject (motion blur), bad focusing (defocusing blur), or a number of other mechanisms which are well modeled by a convolution. In last PDE method is compared with the existing restoration techniques such as weiner filters, median filters [2] and the results are compared on the basis of calculated PSNR for various noises
\end{abstract}

Keywords: PDE,PSF,Deblurring, Weiner filter

\section{INTRODUCTION}

Images are produced in order to record or display useful information. Due to imperfections in the electronic or photographic medium, the recorded image often represents a degraded version of the original scene. The degradations may have many causes, but two types of degradations are often dominant: blurring and noise. The restoration and enhancement of the blurred and noised images are of fundamental importance in image processing applications. To find the original image the degraded images has to be deblurred. The field of image deblurring is concerned with the reconstruction or restoration of the uncorrupted image from a distorted and noisy one. The restoration (deblurring) of images is an old problem in image processing, but it continues to attract the attention of researchers and practitioners. A number of real-world problems from astronomy to consumer imaging find applications for image restoration algorithms. Image restoration is an easily. visualized example of a larger class of inverse problems that The degradation, of an image can be caused by many factors. The movement during the image captures process, by the camera or, when long exposure times are used, by the subject. The out-of-focus optics, use of a wide-angle lens, atmospheric turbulence, or a short exposure time, which reduces the number of photons captured. The confocal microscopy is an optical imaging technique. It enables the reconstruction of 3-D structures from the obtained images.An ideal camera or recording device would record an image so that the intensity of a small piece (pixel) of the recorded image was directly proportional to the intensity of the corresponding section of the scene being recorded. The real cameras violate this model in two ways. First, the recorded intensity of a pixel is related to the intensity in a larger neighborhood of the corresponding section of the scene. This effect in visual images is called blurring. Second, the recorded intensities are contaminated by random noise. The Noise is a unwanted or undesirable information that contaminates an image. Noise appears in images from a variety of sources. First, the digital image acquisition process, which converts an optical image into continuous electrical signal that is then sampled, is the primary process by which noise appears in digital image. The image noise is a random variation of brightness or color information in images produced by the camera. There are fluctuations caused by natural phenomena that add a random value for a given pixel. A blurred or degraded image can be approximately described by this equation

$$
\mathrm{k}=\mathrm{H}^{*} \mathrm{f}+\mathrm{n},
$$

Where the $\mathrm{k}$ is the blurred image, the $\mathrm{H}$ is the distortion operator also called the point spread function(PSF), $\mathrm{f}$ is the original true image, $\mathrm{n}$ is the additive noise, introduced during 
image acquisition, that corrupts the image. The figure shown represents the PSF, point spread function

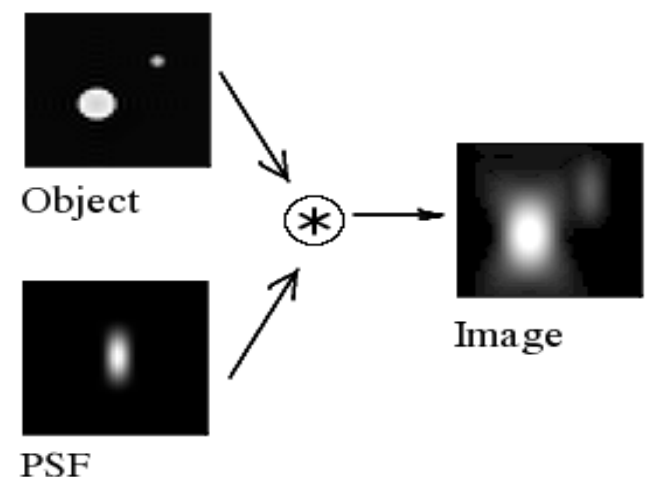

Figure:1 Degradation in image by PSF

The degraded images are deblurred using the traditional techniques .

\subsection{Weiner Filter}

The method is founded on considering image and noise as random process and objective is to find an estimate of deblurred image of the uncorrupted image such that mean square error between them is minimized.The simplest approach is to restore the original image simple by dividing the transform of degraded image by degradation function.

$\mathrm{F}^{\prime}(\mathrm{u}, \mathrm{v})=\mathrm{F}(\mathrm{u}, \mathrm{v})+\mathrm{N}(\mathrm{u}, \mathrm{v}) / \mathrm{H}(\mathrm{u}, \mathrm{v})$

These are the frequency transform of deblurred image,original image,noise density and degraded function

\subsection{Order Statistics Filters}

These are the spatial filters [4] whose response is based on ordering of the pixels contained in the image area and compassed by the filter. The response of the filter at any point is determined by ranking result.

$\mathrm{F} 1(\mathrm{x}, \mathrm{y})=\operatorname{median}\{\mathrm{g}(\mathrm{s}, \mathrm{t})\}$

$\mathrm{F} 1(\mathrm{x}, \mathrm{y})=\max \{\mathrm{g}(\mathrm{s}, \mathrm{t})\}$

$\mathrm{F} 1(\mathrm{x}, \mathrm{y})=\operatorname{mean}\{\mathrm{g}(\mathrm{s}, \mathrm{t})\}$

\section{PURPOSED METHOD}

Image restoration is the pre-processing method that targets to suppress degradation using knowledge about its nature. Restoration attempts to recover an image that has been degraded using a priori knowledge of the degradation phenomenon. Hence, the restoration techniques are focussed towards modelling the degradation and applying the inverse process in order to recover the original image. The relative motion between the camera and the object may lead to blurring of image during its formation on the film of the camera.The travelling wave de-blurring method is a approach that works in spatial domain but the mathematical model discussed in this paper is not generalized and discretization issues and stability criteria of differential equation has not been addressed. In fact, when the proposed differential equation is discretized using forward differencing scheme is unconditionally unstable which may not produce the desired results. A generalized PDE [3] based image model is proposed to model the phenomenon of blurred image formation due to relative motion between camera and the object and further the recovery of original image in spatial domain. Lax scheme is used to discretize the resulting PDE which is mathematically stable and produces good result. Therefore, with the use of Lax method for discretizing the proposed PDE that was initially a flux conservative equation transforms to a ID flux conservative equation with an added diffusion term which is in the form of Navier-Stokes equation. The, additional diffusion term contributes towards further smoothing of image. Let vector $\frac{X}{R^{n}, f: R^{n} \longrightarrow R_{\text {and }}}$ $\underline{\boldsymbol{X}}=\left(\boldsymbol{x}_{1}, \boldsymbol{x}_{2}, \boldsymbol{x}_{3}, \cdots \cdots, \boldsymbol{x}_{n}\right)$ and $\mathrm{f}$ is a function of $\underline{X}$. For $1 \mathrm{D}$ object $f(\underline{X})=x$ and for 2D object i.e. images $f(\underline{X})=(x, y)$. Let $\underline{V}$ represents the velocity vector of object and $\underline{V}=\left(\boldsymbol{v}_{1}, v_{2}, \ldots \ldots, v_{n}\right)$.If object is moving in horizontal direction only then velocity reads as $\underline{V}=v_{x}$ and if object is under motion in XY-space in both horizontal and vertical directions then velocity vector reads as $\underline{\boldsymbol{V}}=\left(\boldsymbol{v}_{x}, \boldsymbol{v}_{y}\right)$. If n-dimensional object $f(\underline{X})_{\text {keeps a }}$ linear uniform motion at a rate $\underline{V}$ in n-Dim space under the surveillance of a camera. The total exposure $g(\underline{X}, t)$ at any point of the recording medium(e.g., film) is obtained by integrating the instantaneous exposure over the time interval $0<=\mathrm{t}<=\mathrm{T}$ during which camera shutter is open. After discretization using Navier-Strokes equation, we get Observed object for duration $\mathrm{T}$ can be modeled as $g(\underline{X}, t)=\int_{0}^{T} f(\underline{X}-\underline{V t}) d t$ 
$g_{j}^{n+1}=g_{j}^{n}-(v \Delta t) \frac{\partial g}{\partial x}+\frac{(\Delta x)^{2}}{2} \frac{\partial^{2} g}{\partial x^{2}}$

From above derived equation the PDE equation is

$I t=I t-(v \Delta t) \frac{\partial g}{\partial x}+\frac{(\Delta x)^{2}}{2} \frac{\partial^{2} g}{\partial x^{2}}$

\subsection{Algorithm for implementing vertical deblurring:}

The Algorithm for this scheme is as follows:-

1. Read the original image $s$ of size $m x n$.

2. Introduce the motion blur in y direction to get $s(y, x, t)$ or we can directly have the blurred image $s(y, x, t)$.

Id $=\mathrm{s}(\mathrm{y}, \mathrm{x})$ : Initial Image

3. Set $d y=0.1, d t=0.1$

4. for $\mathrm{t}=1: \mathrm{n}$ iterations

$\mathrm{Id}=\mathrm{Id}-(\mathrm{v} \Delta \mathrm{t}) \frac{\partial s}{\partial y}+\frac{(\Delta y)^{2}}{2} \frac{\partial^{2} s}{\partial y^{2}}$

// Evolves the sol. after $\mathrm{n}$ iterations end

5. Display the image

\subsection{The Combined Deblurring Algorithm:-}

1. Read the original image $\mathrm{K}$ of size $\mathrm{mxn}$.

2. Filter the image $\mathrm{K}$ to Produce blurred version $\mathrm{h}(\mathrm{x}, \mathrm{y})$ by introducing motion in $\mathrm{x}$-direction.

3. Filter $K(x, y)$ to get final version $K(x, y)$ by introducing motion blur in $\mathrm{y}$-direction $\mathrm{K}(\mathrm{x}, \mathrm{y})$ is the final blurred image with motion introduced in both $\mathrm{x}$ and $\mathrm{y}$ directions).

Initial Image $\mathrm{I}=\mathrm{K}(\mathrm{x}, \mathrm{y})$

4. Set $d x=0.1$

$\mathrm{t}=0.1$, no_iterations $=50,{ }^{\mathrm{V}_{\mathrm{x}}}=1$

5. For $\mathrm{t}=1$ : no_iterations

$\mathrm{I}=\mathrm{I}-\left(\mathrm{v}_{\mathrm{X}} \Delta \mathrm{t}\right) \frac{\partial \mathrm{K}}{\partial \mathrm{x}}+\frac{(\Delta \mathrm{dx})^{2}}{2} \frac{\partial^{2} \mathrm{~K}}{\partial \mathrm{x}^{2}}$

6. $\mathrm{R}=\mathrm{I}$

7. Set $\mathrm{dy}=0.1 \mathrm{dt}=0.1$, num_iterations $=50, v_{y}=1$

8. For $\mathrm{t}=1$ : num_iterations

9. $\mathrm{R}=\mathrm{R}-\left(\mathrm{v}_{\mathrm{y}} \Delta \mathrm{t}\right) \frac{\partial \mathrm{I}}{\partial y}+\frac{(\Delta \mathrm{y})^{2}}{2} \frac{\partial^{2} \mathrm{I}}{\partial \mathrm{y}^{2}}$
10. Get $\mathrm{R}$ and display as final deblurred Image

\section{RESULTS}

The blurring of images can be caused by movement of object or camera while capturing the image. The deblurring of Images is the reconstruction or restoration of the uncorrupted image from a distorted and noisy one. In this paper, an idea for two directional image deblurring algorithm is introduced which uses basic concepts of PDEs having the prior knowledge about the PSF. Motion Blurring is introduced in two directions: horizontal and vertical. Then we proposed PDEs based model for image deblurring considering both the directions which is based on the mathematical model. A simple two dimensional algorithm has been introduced and implemented. The results show better quality of images by applying this algorithm compared to the previously designed techniques. The results are compared on the basis of PSNR calculated for the several noises such as Gaussian noise, salt and pepper noise, speckle noise etc. The deblurring is done for the mean taken as 0 and variance is 0.001 for all the noises. The results shown below for the Gaussian noise deblurred by the various filters and is shown that PSNR is better for the PDE method.

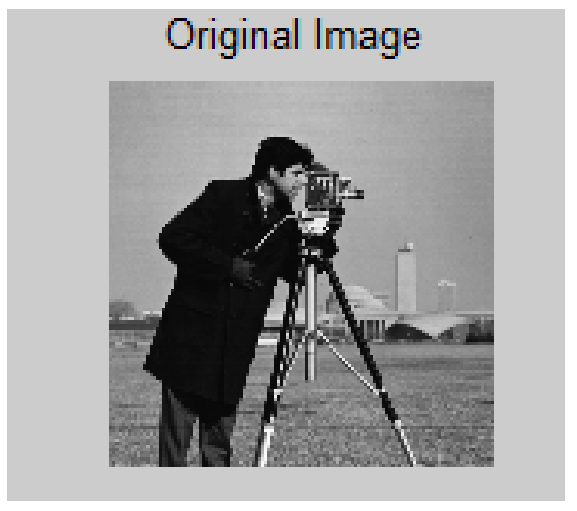

Figure: 2 Original image

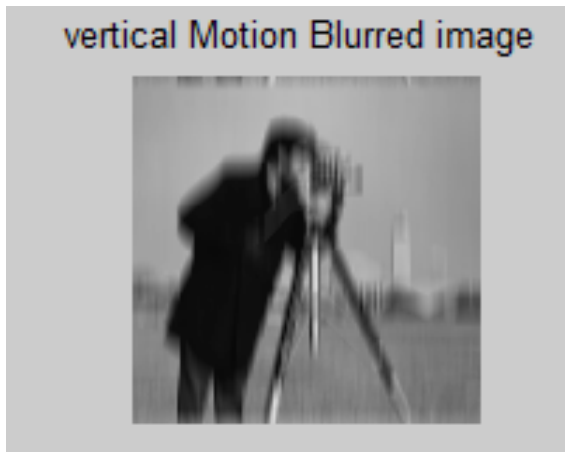

Figure: 3 Image blurred in Y direction 
International Journal of Computer Applications Technology and Research Volume 2- Issue 3, 232 - 236, 2013, ISSN: 2319-8656

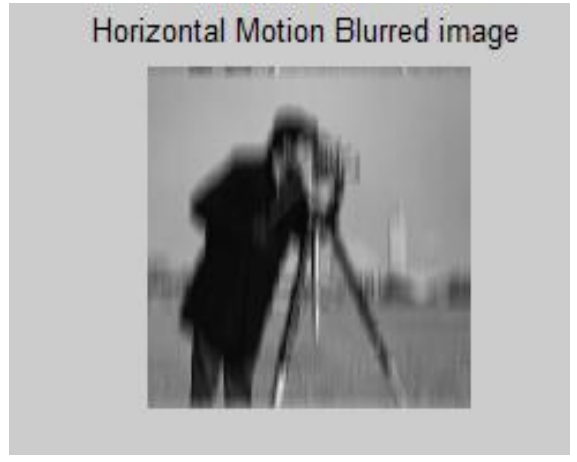

Figure: 4 Image blurred in $\mathrm{X}$ direction

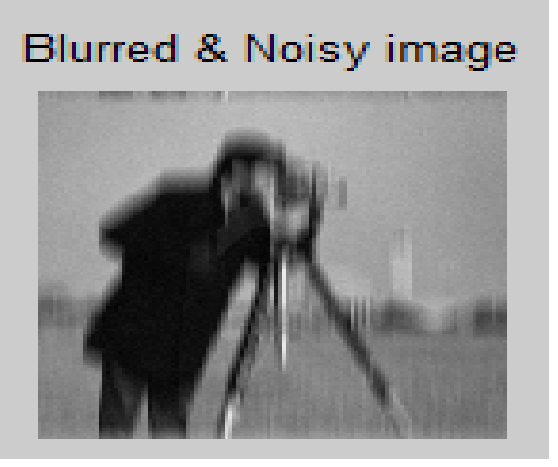

Figure: 5 Noise added in blurred image

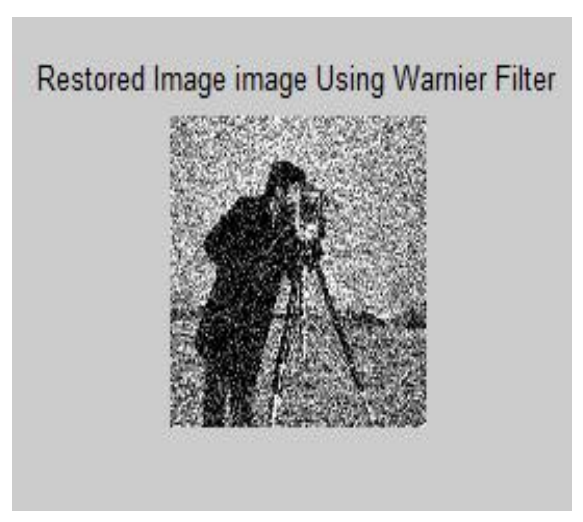

Figure: 6 Deblurred image in Y-direction

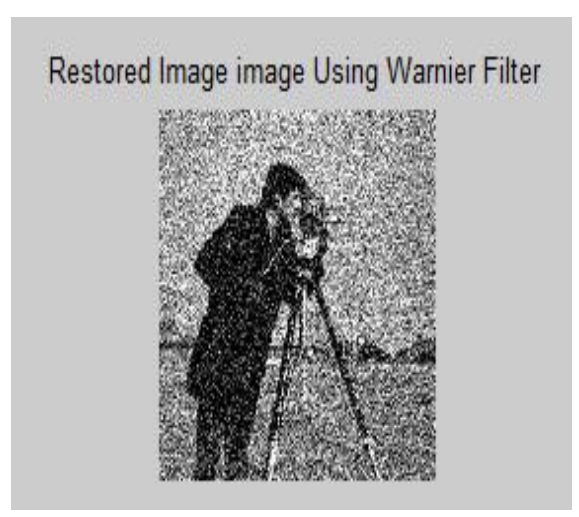

Figure: 7 Deblurred image in X-direction

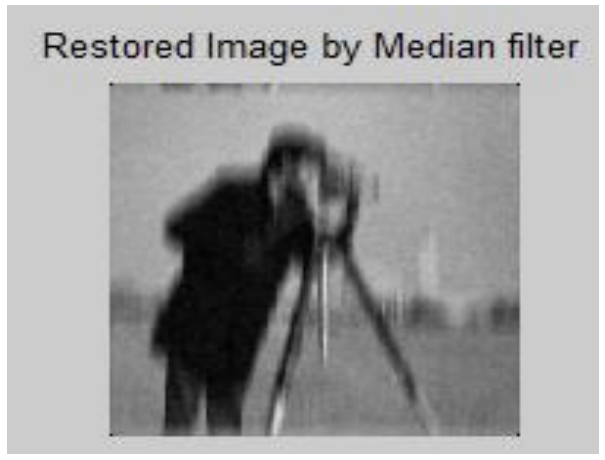

Figure: 8 Deblurred image in Y-direction

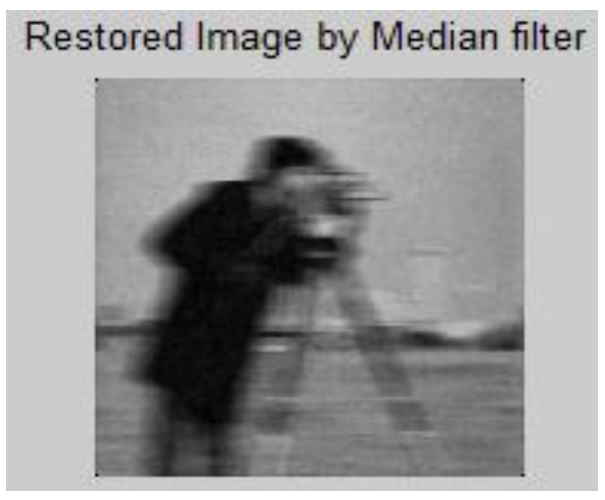

Figure:9 Deblurred image in X-direction

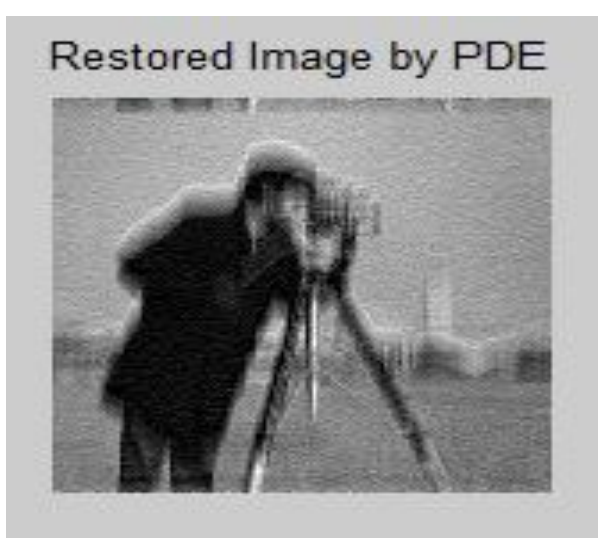

Figure:10 Deblurred image in Y-direction

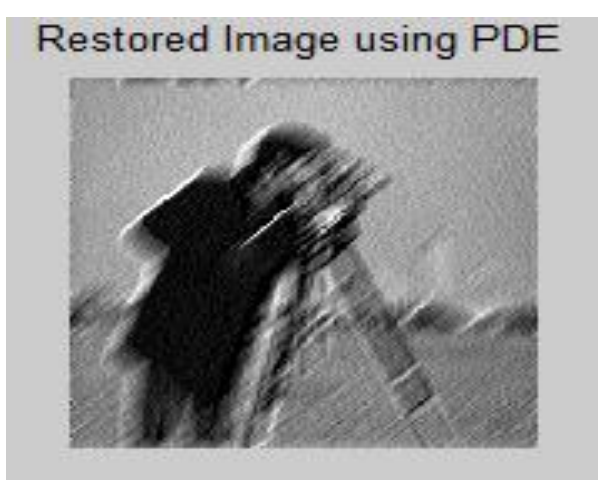

Figure: 11 Deblurred image in X-direction 


\subsection{PSNR Table:}

The PSNR based comparison is done among the different techniques.PSNR Table is calculated for different techniques and for several noises and is shown that PDE shows better results.

Table1: PSNR calculation for different techniques.

\begin{tabular}{|c|c|c|c|}
\hline Noise type & Blurr & Technique & PSNR \\
\hline Gaussian & Vertical & MedianFilters & 28.1294 \\
\hline Gaussian & Vertical & Wiener Filter & 14.9225 \\
\hline Gaussian & Vertical & PDE & 40.1383 \\
\hline Impulse & Vertical & MedianFilters & 36.9146 \\
\hline Impulse & Vertical & Wiener Filter & 8.8892 \\
\hline Impulse & Vertical & PDE & 24.855 \\
\hline Poisson & Vertical & MedianFilters & 23.3081 \\
\hline Poisson & Vertical & Wiener Filter & 9.7698 \\
\hline Poisson & Vertical & PDE & 26.9374 \\
\hline Speckle & Vertical & MedianFilters & 19.5736 \\
\hline Speckle & Vertical & Wiener Filter & 7.2725 \\
\hline Speckle & Vertical & PDE & 19.7322 \\
\hline
\end{tabular}

\section{ACKNOWLEDGMENTS}

Ablend of gratitude, pleasure and great satisfaction is what I feel to convey my indebtedness to all those who directly or indirectly contributed to the successful publication of this paper. I express my profound and sincere gratitude to my Guide, Mr.Puneet Sharma, A.P in CSE department, whose Persistence guidance and support helped me in the successful completion of the paper in stipulated time. His expert knowledge and scholarly suggestion help me a lot. I am grateful to $\mathrm{Mr}$. Neeraj Gupta, HOD, CSE, HCE Sonepat for his support. I am thankful to all my Professors and Lecturers and members of the department for their generous help in various ways for the completion of this work.

\section{REFERENCES}

[1] M. Bertero and P. Boccacci," Introduction to the Inverse Problems in Imaging," IOP Pub., Bristol, UK, 1998.

[2] Alliney, S.: Recursive median filters of increasing order:variational approach. IEEE Transactions on Signal rocessing 44(6), 1346-1354 (1996).
[3] Rajeev Srivastava, Harish Parthasarthy, JRP Gupta and D. Roy Choudhary, "Image Restoration from Motion Blurred Image using PDEs formalism", IEEE International Advance Computing Conference (IACC 2009), March 2009.

[4] www.google.com 